\title{
Pre-Conscious Assessment of Trust: A Case Study of Financial and Health Care Web Sites
}

\author{
William Albert, William Gribbons, \& Jindrich Almadas, Bentley University
}

\begin{abstract}
The goal of this study was to determine the reliability of pre-conscious trust assessments of web sites. Participants in Experiment $1(\mathrm{n}=72)$ viewed 50 screenshots of popular financial and health care home pages in a random order in two separate trials. Each screenshot was presented for $50 \mathrm{~ms}$, followed by a mask for $150 \mathrm{~ms}$, followed by an assessment of trust on 9-point semantic differential scale from distrust to trust. Results from a series of Pearson Product Moment Correlations showed that approximately one-half of the participants were consistent in their trust assessments for the same web sites across trials. The correlation between trust assessments on the first and second trials, averaged across all participants was statistically significant $(\mathrm{p}<.001)$. Results from Experiment $2(\mathrm{n}=11)$, utilizing a different method for participant participation, showed a similar pattern of results. These findings suggest that pre-conscious mind plays a more significant role in assessing trust than previously believed.
\end{abstract}

\section{INTRODUCTION}

Trust is a critical component of human relationships and the relationships facilitated by technology-based services. At its most basic level, Corritore \& Wiedenbeck (2003) describe trust as a "social lubricant" or "social capital" essential for cooperative behavior between people (738). Trust in technology-mediated experiences has garnered close scrutiny over the past decade corresponding with the growth of electronic financial transactions and the delivery of sensitive electronic information through healthcare and government services. In each case, the user must not only trust the integrity of the sponsoring party but also the electronic experience.

To date, most research has focused on the conscious elements of the trust relationship. Although considerable research exists examining the pre-attentive assessment of other emotions, the trust emotion has largely been ignored. As it relates to the larger affective system, research examining the preconscious experience confirms that some emotions are registered rapidly and once registered build in the subconscious mind and ultimately affect the larger conscious experience. Previous research has examined the preconscious experience of simple emotions: good-bad and positive-negative (Norman, 2004b). More recent studies have examined more complex assessments such as the attractiveness of faces (Olson and Marshuetz, 2005) and web sites (Lindgaard, Fernandes, Dudek, \& and Brown, 2006). Our three-part study builds on the work of Lindgaard et al (2006) and Olson and Marshuetz (2005) to examine whether trust is assessed rapidly in the preconscious mind in response to yet unknown visual elements.

While much is known about trust and its effects on the conscious experience, little is known about when and how it might be triggered in the preconscious mind. Norman (2004a) suggests that any trust existing in the technology experience is largely the product of chance or happenstance. In stage one of our study, we will examine the reliability of a rapid trust assessment. In stage two, we will compare trust assessments on pre-attentive and conscious levels. In stage three, we will attempt to trace any effect to design attributes. Finally, in stage four, we will examine the effects of trust/distrust primes on subsequent cognitive acts. It is the goal of this investigation to first confirm the preconscious experience of trust and in later studies to identify design elements that reliably trigger trust and move the design of a trustworthy experience beyond the likelihood of "chance." This paper reports on stage one.

\section{WHAT IS TRUST?}

Despite the diverse range of contributions from equally diverse disciplines, the vast majority identify attributes registered at a conscious or reflective level. Corritore and Wiedenbeck (2003) proposed two broad types of trust: cognitive and emotional. Cognitive trust requires rational reasons why this object merits trust while emotional trust is motivated by strong positive feelings. Kim and Prabhakar (2000) consider the views of sociologists and economists that examine "how institutions are established and incentives are used to reduce uncertainty associated with transactions among relative strangers (538). Consistent with the uncertainty theme, McKnight (2002) includes the predictability of the other party in his perception of trust. Similarly Fogg (2003b) highlights the importance dependability of the person, object or process in his discussion of trust. Norman (2004a) and Corritore and Wiedenbeck (2003) stress the importance of confidence and meeting expectations in their definitions of trust. Norman (2004a) aligns with Belanger, Hiller, and Smith (2002) and Fogg (2003b), highlighting the importance of integrity and reliability in their definition. While the terms vary dependability, predictability, and reliability -- the underlying concept is remarkably similar. Riding, Gefen, and Arinze (2002) and McKnight (2002) concurs on the role of integrity in the trust relationship. Riding, Gefen, and Arinze (2002), McKnight et al. (2002) and Fogg (2003b) stress the importance of ability and expertise in their views of trust.

One the most dominant and reoccurring themes in research related to trust is the role of risk -- without risk there is no need for trust (Corritore, C. K., \& Wiedenbeck, S., 2003 and 
McKnight et. al., 2002). Similarly, Kim and Prabhakar (2000) defined trust as an individual's reliance on another party under conditions of dependence and risk. Considering that risk is a function of the probability that a hazard arises and the consequences of the hazard (537).

B.J. Fogg (2003a), founder of Stanford Persuasive Technology Lab, identifies four elements of credibility: presumed: assumptions in the mind of the perceiver; surface: simple inspection or initial firsthand experience; reputed: third party endorsements; earned: reputation built over time (130). Like so many of the other perspectives, each of these elements operates at some level of consciousness. Norman (2004a) also draws a distinction between trust in a person and trust in a system. In the latter, he emphasizes the importance of the system performing precisely according to expectations (142). Wakefield (2004) breaks out a separate category called "initial trust" which is "characterized by lack of experience with or firsthand knowledge of the other party or a new technology (p. 95). However, Wakefield limits his examination of factors that build initial trust on a web site to conscious, cognitive factors such as communication, opportunism, product brand quality, and web site attractiveness. Focusing on trust in virtual communities, Riding, Gefen, and Arinze (2002) define trust as a "set of beliefs that the other party will refrain from opportunistic behavior and will not take advantage of the situation," (275)

\section{PRE-CONSCIOUS ASSESMENT OF AFFECT AND VISUAL PRIMITVES}

As discussed previously, traditional discussion of trust has largely focused on conscious assessment of information, source, and channel. Little research exists that examines preconscious elements of trust. The purpose of this study is not to challenge the conscious elements of the trust relationship but to extend our understanding of trust by defining an initial discrimination of trust at the preconscious level. Generally speaking, any target processed at rates quicker than $250 \mathrm{~ms}$, the speed of a fixation, is considered preattentive. Should this primitive foundation exist, it would add yet another layer to the trust experience and have significant implications for system design.

\section{METHODS}

The goal of the study is to determine the reliability of preattentive trust assessments. This is achieved by briefly presenting visual stimuli in the form of popular financial and health-related web site home pages and examining the consistency of responses between participants' trust assessments across multiple exposures.

\section{Participants:}

72 participants took part in the study. Participants were recruited through Utest, a panel of professional software testers. Limited demographic data were collected on a subset of participants $(n=21)$. The average age is 31 , and $76 \%$ are male. Participants reside in the US, UK, Canada, India, and The Netherlands. All participants were compensated $\$ 10$ for their involvement in the study. Because this experiment involved pre-conscious assessments, participant's professional and academic background was not a concern.

\section{Apparatus}

The study was conducted using Inquisit by Millisecond Software. This software allows individuals to participate in controlled experiments using their own computer in any location, provided they have Internet access. All stimuli are loaded directly on to the participant's computer prior to the start of the experiment to ensure consistent performance.

\section{Stimuli}

The stimuli consist of a total of 50 screenshots of web site home pages. 25 screenshots are the home pages of financial web sites; 25 screenshots are the home pages of health-related web sites. Web sites represent the top 25 health care and financial services companies from the Fortune 1000 list of America's largest corporations. The screenshots were captured at a $1280 \times 768$ resolution.

\section{Design}

The study was set up as a within-subject design, whereby each participant received the same set of screenshots across two trials. In the first trial, 50 screenshots were presented in a random order. In the second trial, the same set of 50 screenshots was presented in a new random order.

\section{Procedure}

Participants took part in individual sessions. Participants accessed the study through their own web browser, at which point they were given a brief description of the study and agreed to participate. Participants were instructed to view each image, and provide an assessment of trust along a semantic differential scale from 1 (distrust) to 9 (trust). Based on pilot testing, we knew some participants would have difficulty assessing trust after an extremely short exposure (50ms). As a result, we suggested participants rely on their initial "gut feel" even if it is just a guess, as opposed to deeper contemplation.

Each screenshot was presented for $50 \mathrm{~ms}$, followed by a mask for $150 \mathrm{~ms}$ (see Figure 1). Displaying each screenshot for $50 \mathrm{~ms}$ is consistent with Lindgaard et al. (2006). From the participant's perspective, each screenshot was just barely more than a flash of light. There was a one second delay after each trust assessment and presentation of the next screenshot (see Figure 1). After the first 50 screenshots, participants were given an opportunity for a break before completing the second set of 50 screenshots. The total time to complete the study was approximately 15 minutes. 


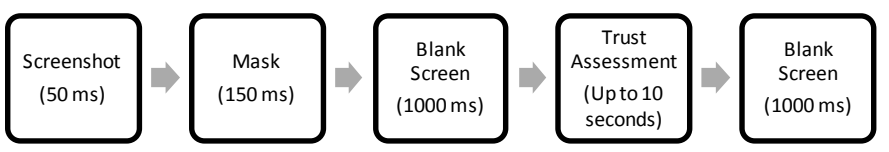

Figure 1. Sequence of stimuli for each screenshot.

\section{EXPERIMENT 1}

Out of 72 participants, 8 were not included in the analysis due to their response patterns. All of the removed participants provided the same consecutive response for more than $50 \%$ of the screenshots. For example, a participant may have entered a "9" for their level of trust for 80 consecutive screenshots. This type of response pattern strongly suggests strong satisficing behavior, common when participants are taking part in an online study and have a strong desire to receive the incentive, while providing minimal effort. This concern is addressed in Experiment 2.

The reliability of trust assessments was first evaluated by examining the proportion of participants who reached a statistically significant correlation between their responses in trials 1 and 2. Table 1 shows that approximately one half $(48.4 \%)$ of participants showed a significant correlation in their responses between trials at the $\mathrm{p}<.05$ and $\mathrm{p}<.01$ levels. An additional $7.8 \%$ of participants $(n=5)$ had a marginally significant correlation, $\mathrm{p}<0.10 .37 .5 \%(\mathrm{n}=24)$ of the Pearson Product Moment correlation coefficients (r) were less than 0.20 , and $76 \%(\mathrm{n}=49)$ were less than 0.40 (see Table 2). $17.2 \%$ of participants $(n=11)$ had a correlation coefficient exceeding 0.50 .

Table 1. Significance levels of each correlation coefficient for all participants in Experiment 1

\begin{tabular}{|l|r|r|}
\hline Significance & \# of Participants & \% of Participants \\
\hline$p<.01$ & 21 & $32.8 \%$ \\
\hline$p<.05$ & 10 & $15.6 \%$ \\
\hline$p<.10$ & 5 & $7.8 \%$ \\
\hline$p>.10$ & 28 & $43.8 \%$ \\
\hline
\end{tabular}

Table 2. Distribution of correlation coefficients for all participants in Experiment 1

\begin{tabular}{|l|r|r|}
\hline $\begin{array}{l}\text { Correlation } \\
\text { Coefficient }\end{array}$ & \# of Participants & \% of Participants \\
\hline$<.10$ & 11 & $17.2 \%$ \\
\hline $.10-.19$ & 13 & $20.3 \%$ \\
\hline $.20-.29$ & 12 & $18.8 \%$ \\
\hline $.30-.39$ & 13 & $20.3 \%$ \\
\hline $.40-.49$ & 4 & $6.3 \%$ \\
\hline $.50-.59$ & 6 & $9.4 \%$ \\
\hline $.60-.69$ & 3 & $4.7 \%$ \\
\hline $.70-.79$ & 1 & $1.6 \%$ \\
\hline $.80-.89$ & 1 & $1.6 \%$ \\
\hline $.90-1.0$ & 0 & $0.0 \%$ \\
\hline
\end{tabular}

Similar to Lindgaard et al (2006), we examined the average trust assessment for the 50 home pages. Each data point represents the average trust assessment based on all participants for one screenshot, for each trial (see Figure 2). The Pearson Product Moment Correlation Coefficient (r) was $0.81(\mathrm{p}<.001)$, indicating a significant association between trials 1 and 2 . The $\mathrm{R}^{2}$ value corresponds to .66, indicating that two-thirds of the variance in trial 2 is explained by trial 1 . It is interesting to note that even though these 50 home pages represent the top health-care and financial services companies in the US, presumably with access to professional web designers, there is considerable variability in perceived trust.

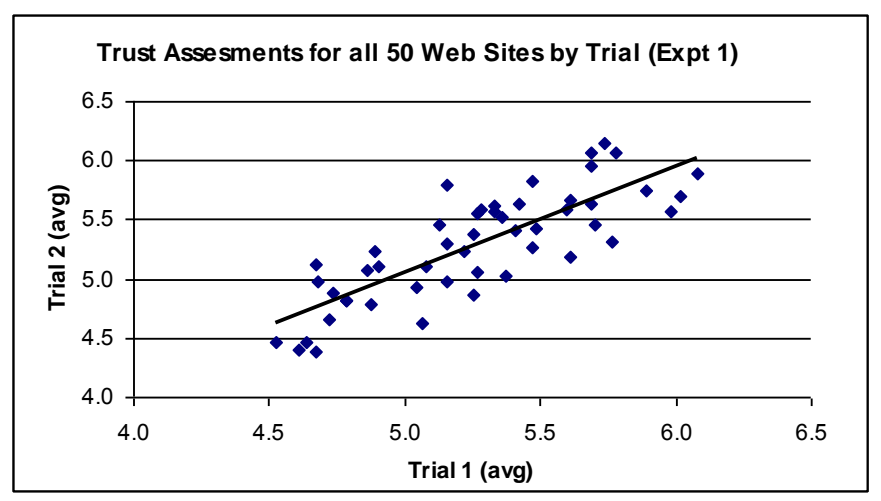

Figure 2. Average trust assessments for trials 1 and 2 for all 50 home pages, averaged across all participants in Experiment 1.

Overall, the data from Experiment 1 suggest that trust is processed at a pre-attentive level, at least with some participants. Roughly one half of the participants showed a statistically significant consistency between how they rated the same screenshots across trials. Furthermore, there was a highly significant correlation between trials for the average trust assessment ratings for the 50 screenshots.

\section{EXPERIMENT 2}

A concern of Experiment 1 was possible disengagement by some of the participants. $11 \%$ of the participants $(n=8)$ were removed from the analysis due to likely satisficing behavior. In Experiment 2 we aimed to reduce the likelihood of satisficing behavior by running the same experiment in a more controlled environment. If we find a roughly comparable set of results we can feel confident that the methods used in Experiment 1 provided valid data.

Eleven graduate students from Bentley University participated in the study, using the identical methods as Experiment 1, with the exception that participants were in a class room setting.

\section{Results}

Seven out of the $11(63.6 \%)$ participants achieved a significant correlation of their trust responses between trials 1 and 2 (see Table 3). This compares to roughly half of the participants in Experiment 1 that reached a significant correlation between 
their responses in the first and second trials (see Table 1). A Chi-Square test comparing the distribution of participants in both experiments was not statistically significant, $(\mathrm{p}=.45)$, suggesting that there are no significant differences in the individual results from Experiment 1 and Experiment 2. The Pearson Product Moment Correlation Coefficients (r) ranged from 0.18 to .68 , with five out of the 11 participants having an r-value greater than 60 .

Table 3. Significance levels of each correlation coefficient for all participants in Experiment 2

\begin{tabular}{|l|r|r|}
\hline Significance & \# of Participants & \% of Participants \\
\hline$p<.01$ & 7 & $63.6 \%$ \\
\hline$p<.05$ & 0 & $0.0 \%$ \\
\hline$p<.10$ & 1 & $9.1 \%$ \\
\hline$p>.10$ & 3 & $27.3 \%$ \\
\hline
\end{tabular}

An examination of the average trust assessments for the 50 screenshots revealed a Pearson Product Moment correlation coefficient (r) equal to $0.76, \mathrm{p}<.001$, with an $\mathrm{R}^{2}$ value of .54 . This is comparable to the results in Experiment $1, \mathrm{r}=0.81, \mathrm{R}^{2}$ $=.66$.

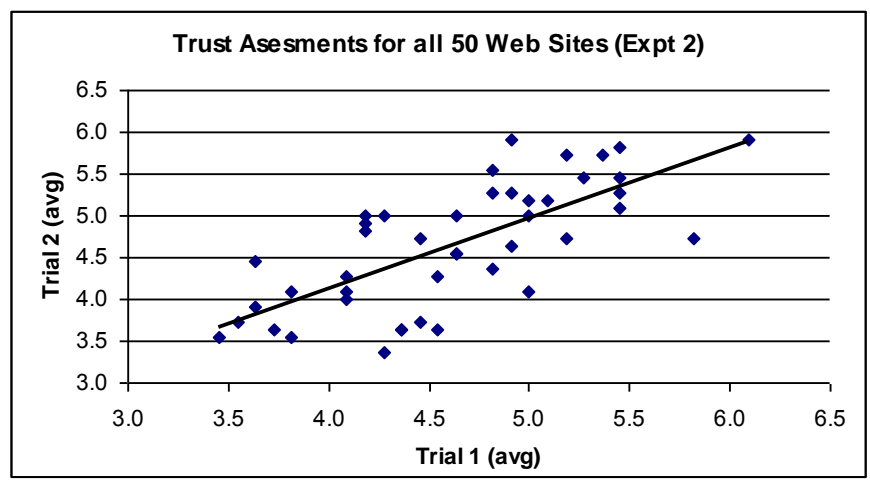

Figure 2. Average trust assessments for trials 1 and 2 for all 50 home pages, averaged across all participants in Experiment 2.

Results of Experiment 2 are comparable to Experiment 1. Roughly half of the participants achieved a statistically significant level of consistency of trust assessments between the two trials in both experiments. The correlation coefficients of the 50 screenshots was also comparable, $r=.81$ in Experiment 1, and $r=.76$ in Experiment 2, both significant at the $\mathrm{p}<.001$ level. Therefore, it appears as though collecting data remotely (Experiment 1) or in-person (Experiment 2) produces a consistent set of results.

\section{CONCLUSIONS}

The results of Experiments 1 and 2 suggest that individuals are capable of processing trust on a pre-attentive level. Approximately one-half of the participants in Experiment 1, and two-thirds of participants in Experiment 2 demonstrated a consistent level of trust assessments. Furthermore, in both experiments there was a significant correlation between trust assessments in the first and second trial when averaging across all participants. If individuals were not able to process trust on a pre-attentive level, there would not have been any level of consistency between trials. This was clearly not the case.

This finding builds upon the work of Lindgaard et al. (2006) by demonstrating that not only is visual attractiveness of web sites pre-attentively processed, but also trust. Taken together, this research suggests that the preconscious mind may play a far more critical role in our conscious decision making and judgments than previously believed. This has significant implications for the design of not only web sites, but any technology that requires some level of trust of the user. Designers should be keenly aware of how visual primitives such as color, closure, and size interact to influence trust assessments on a pre-attentive level. Simply focusing on content to relay the notion of trust may not be enough.

Stage two of our research will test the reliability of preattentive trust assessments by comparing trust assessments on pre-attentive and conscious levels. Stage three will isolate specific visual attributes to identify the drivers of trust (and distrust). By doing this, we will be able to provide a set of guidelines on how to design technology to enhance the trust relationship between user and technology. In stage four we will focus on how pre-attentive processing manifests itself in conscious decision making and judgments. Are any biases about trust developed at a pre-attentive level easily dismissed at the conscious level as more information is acquired, or are these biases a powerful force in shaping judgments and decisions?

\section{REFERENCES}

Belanger, F., Hiller, J., \& and Smith, W. (2002). Trutworthiness in electronic Commerce: The Role of privacy, Security, and Site Attributes. Journal of Strategic Information Systems , 11, 245-270.

Corritore, C. K., \& Wiedenbeck, S. (2003). Online Trust: Concepts, evolving themes, a model. International Journal of Human-Computer Studies, 58, 737-758.

Fogg, B. (2003a). Persuasive Technology. San Francisco: Morgan Kaufmann.

Fogg, B. (2003b). Prominence-Interpretation Theory: Explaining How People Assess Credibility Online. ACM CHI 2003: New Horizons, (pp. 722-723). Ft. Lauderdale, Florida.

Kim, K. and Prabhakar, B.. (2000). Initial Trust, Perceived Risk, and the Adoption of Internet Banking, Proceedings of the twenty first international conference on Information systems. International Conference on Information Systems, (pp. 537 - 543 ). Brisbane, Queensland, Australia.

Lindgaard, G., Fernandes, G., Dudek, C., \& and Brown, J. (2006). Attention Web Designers: You have 50 milliseconds to make a good first impression! Behavior and Information Technology, 25 (2), 115-126. 
McKnight, H. C. (2002). The Impact of Initial Consumer Trust on Intentions to Transact With a Web Site: A Trust Building Model. Journal of Strategic Information Systems, 11, 297-323.

Norman, D. (2004a). Emotional Design. New York: Basic Books.

Norman, D. (2004b). Introduction to the Special Section on Beauty, Goodness, and Usability. Human Computer Interaction, 19, 311-318.

Olson, I. and Marshuetz, C. (2005). Facial Attractiveness Is Appraised in a Glance. Emotion, 5 (2), 498-502.

Riding, C., D., G., \& B., A. (2002). Some antecedents and effects of trust in virtual communities. International Journal of Strategic Information Systems, 11, 271-295.

Wakefield, R., Stocks, M., \& and Wilder, M. (2004). The Role of Web Site Characteristics in Initial Trust Formation. The Journal of Computer Information Systems, 45 (1), 94-104.

\section{ACKNOWLEDGEMENT}

The authors wish to acknowledge the generosity of UTest for providing access and compensation to participants in Experiment 1, and thank the Graduate students at Bentley University who participated in this study. 